\title{
BASES NEUROFISIOLÓGICAS DEL APRENDIZAJE COGNITIVO SOCIAL
}

EL SISTEMA DE LAS NEURONAS ESPEJO

\section{Juan Francisco del Rosario Nieto \\ Universidad Católica Sedes Sapientiae}

Fecha de recepción: septiembre de 2012 Fecha de aceptación: octubre de 2012

Resumen: El presente ensayo vincula los recientes descubrimientos relacionados con el Sistema de las Neuronas Espejo realizados en el campo de la Neurociencia con la Teoría del Aprendizaje Cognitivo Social formulada por Albert Bandura. Según Van Gog et al. (2009: 21) el descubrimiento de las neuronas espejo es el mayor hallazgo relevante al aprendizaje por observación.

Palabras Clave: espectro autista, aprendizaje cognitivo social, sistema de neuronas espejo

ABSTRACT: This paper links recent findings related to the mirror neuron system made in the field of neuroscience with Social Cognitive Learning Theory by Albert Bandura formulated. According to Van Gog et al. (2009: 21) the

* Juan Francisco del Rosario Nieto es licenciado en Psicología por la Universidad Ricardo Palma. Diplomado en Neuropedagogía por la Asociación Educativa para el Desarrollo Humano - ASEDH, Centro Iberoamericano de Neurociencias, Educación y DesarrolloCEREBRUM y Universidad Católica San Pablo. 
discovery of mirror neurons is the major finding relevant to observational learning.

KeYwords: autism spectrum, social cognitive learning, mirror neuron system

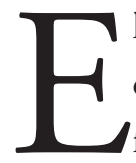

1 ser humano, en toda su complejidad individual y social, ha sido objeto de estudio desde distintos enfoques. Éstos han permitido formular cuerpos sistemáticos de conocimientos que han dado lugar a distintas ciencias. Uno de los aspectos que ha llamado la atención es el comportamiento o conducta humana y dentro de éste un tema específico que ha sido, es y, muy probablemente, será estudiado y analizado es el referido al aprendizaje. Es decir, dilucidar cómo y a través de qué procesos el hombre adquiere conocimientos y destrezas motoras así como las conductas relacionadas con el comportamiento social, moral y ético.

Sobre el particular se han desarrollado, entre otras, un conjunto de aproximaciones teóricas tales como: Condicionamiento Clásico [Pavlov, 1849-1936], Teoría Conexionista o Ensayo-Error [Thorndike, 18741949], Teoría Sistémica de la Conducta [Hull, 1884-1952], Principio de Contigüidad [Guthrie, 1886-1959], Teoría Psicogenética [Piaget, 18961980], Teoría Sociocultural [Vigotsky, 1896-1934], Condicionamiento Instrumental u Operante [Skinner, 1904-1990], Teoría del Procesamiento de la Información [Gagné, 1916-2002], Teoría del Aprendizaje Empírico [Rogers, 1902-1987], Teoría Cognitivista [Bruner, 1915], Teoría de la Asimilación y el Aprendizaje Significativo [Ausubel, 1918-2008], Teoría de la Modificabilidad Cognitiva Estructural y Experiencia de Aprendizaje Mediatizado [Feurerstein, 1921], Teoría del Aprendizaje Social [Bandura, 1925]. 
En 1996, Giacomo Rizzolatti, Leonardo Fogassi y Vittorio Gallese trabajaban en el laboratorio de la Universidad de Parma (Italia); habían colocado electrodos en la corteza inferior de un mono macaco a fin de estudiar qué neuronas especializadas intervenían en el control del movimiento de la mano y específicamente al asir objetos o ponerlos encima de otros. Durante el experimento registraban la actividad de sólo una neurona en el cerebro del macaco. De pronto, observaron que algunas neuronas del mono reaccionaban al momento que uno de los investigadores tomaba un plátano. Entonces, se formularon la siguiente pregunta: ¿cómo es posible que haya sucedido esto si el animal no se ha movido? Al principio se creyó que era un error de medición; pero, constataron que algunas neuronas del cerebro del simio reaccionaban cuando otros sujetos producían los movimientos y éstos eran observados por el macaco. Posteriormente, evidencias obtenidas a partir de datos proporcionados por Imagen por Resonancia Magnética Funcional [IMRf], Estimulación Magnética Transcraneal [TMS, por sus siglas en inglés] y Electro Encéfalo Grama [EEG] llevaron a formular la conjetura que los sistemas de neuronas espejo encontradas en el macaco también estaban presentes en el ser humano.

$\mathrm{Al}$ respecto, Mukamel et. al. (2010: 750) publicaron un informe en el que comunicaban a la comunidad científica que neuronas especiales responden en humanos durante la ejecución y observación de acciones. En dicho informe se señaló que:

En los seres humanos, las mediciones indirectas de la actividad neural fundamentan la existencia de mecanismos espejo sensoriomotores semejantes [a los de los macacos] en las áreas frontales y parietales..., otras regiones motoras... y también la existencia de mecanismos multisensoriales en regiones no 
motoras... Registramos en los cerebros humanos la actividad extracelular de 1177 células en las cortezas frontal medial y temporal mientras que los pacientes ejecutaban u observaban la aprehensión de objetos con la mano y la expresión facial de emociones. (Traducción del autor del ensayo). ${ }^{1}$

Las aproximaciones teóricas formuladas para la explicación del aprendizaje y mencionadas anteriormente ¿̨tienen un correlato neurofisiológico en los procesos neurales que se producen en el cerebro? En especial, la Teoría del Aprendizaje Cognitivo Social formulada por Albert Bandura ¿tiene un correlato neurofisiológico con el reciente descubrimiento de las neuronas espejo por la neurociencia? ¿las neuronas espejo pueden sustentar neurofisiológicamente la teoría del aprendizaje social cognitivo?

Sobre el particular Rondal (2007: 51 y 52) formula la hipótesis siguiente: «Es probable también que dichas neuronas [espejo], constituyan la base, al menos en parte, de la habilidad de la imitación y, por tanto, de la capacidad de aprendizaje por observación».

Teniendo en cuenta que la mayoría de las formulaciones teóricas mencionadas han tenido un fuerte impacto en el ámbito pedagógico, es decir, en la relación profesor-alumno, conceptuado este como buscador y procesador activo de la información ¿en qué medida el descubrimiento de las neuronas espejo y de su vinculación con la teoría del aprendizaje social cognitivo puede aportar a la pedagogía como ciencia? ¿es posible que esa vinculación promueva nuevos procedimientos pedagógicos?

1 In humans, indirect measures of neural activity support the existence of sensory-motor mirroring mechanisms in homolog frontal and parietal areas..., mother motor regions..., and also the existence of multisensory mirroring mechanisms in nonmotor regions... We recorded extracellular activity from 1177 cells in human medial frontal and temporal cortices while patients executed or observed hand grasping actions and facial emotional expressions. 
Cabe destacar que dichas formulaciones teóricas sobre el aprendizaje también han tenido amplia repercusión en el ámbito terapéutico; pero, este aspecto rebasa el objetivo del presente ensayo.

\section{LA TEORÍA DEL APRENDIZAJE SOCIAL FORMULADA POR ALBERT BANDURA}

Algunas de las corrientes de pensamiento mencionadas anteriormente se pueden agrupar en un solo concepto: conductismo. Es decir, explican que la conducta es resultado de un vínculo asociativo entre estímulos y respuestas o respuestas-consecuencias. Por lo tanto, el análisis de la conducta, en términos de comportamiento, sea animal o humano se realiza a partir de la aplicación de métodos experimentales; es decir, la intervención de variables que se pueden observar, medir, manipular, correlacionar; y, dejar de lado todo aquello que se estima subjetivo, lo interior del sujeto y no visible. ¿Qué es lo que determina que el sujeto aprenda según esta concepción teórica? En primer lugar: el o los estímulos; es decir, si presento los estímulos adecuados obtendré las respuestas deseadas; en segundo lugar: el o los reforzadores para establecer la relación entre estímulo presentado y conducta manifiesta. Esto significa que el entorno, lo exterior al sujeto, determina su conducta. Como se puede apreciar es reduccionista y de carácter mecanicista que aporta poco al entendimiento de las conductas complejas del ser humano.

Bandura, también, consideró que dicha explicación era demasiado simple. Por ello, en su autobiografía, Bandura (2006: 8) señaló lo siguiente: 
Hallé este behaviorismo teórico discordante con la realidad de que la mayoría de lo que aprendemos lo hacemos mediante modelos sociales. No podría imaginar una cultura en la que lenguaje, modos, costumbres y prácticas familiares, competencias laborales, prácticas religiosas y políticas se formaran gradualmente en cada uno de sus miembros premiando y castigando las consecuencias de sus ejecuciones, ensayos de intento error.

Esasí que conjuntamente con Richard Waltery Robert Crains iniciaron el estudio de los determinantes familiares de los estilos hiperagresivos de jóvenes que no presentaban una conducta antisocial. Bandura (2006: 8) afirmó que: «Hallamos que los modelos parentales de orientaciones agresivas jugaban un papel en la transmisión familiar de la agresividad». Al respecto, Garrido (2000: 2) comentó sobre Bandura que le:

Atrae su interés el siguiente resultado: los padres niegan la agresividad de sus hijos. Argumentan que en casa se les castiga cuando su comportamiento hiere a los demás. En el marco conductista esto encaja perfectamente. Con mucha probabilidad, infiere nuestro psicólogo, los niños están aprendiendo de sus padres la conducta agresiva y la utilizan en contextos distintos. Los padres, pues, son el modelo a quien imitan los hijos agresivos.

Al respecto, Bandura, en 1965, realizó un experimento con el propósito de estudiar la influencia de los modelos en el comportamiento imitativo infantil con relación a tres situaciones del refuerzo. A tres grupos, conformados por niños y niñas, se les proyectaba una película cuya primera parte era igual para todos; los niños y las niñas observaban cómo un hombre 
adulto golpeaba ferozmente a un muñeco de goma (dominguillo²) y, a la vez, descargaba su agresividad verbal contra el muñeco. La segunda parte era diferente para dos grupos.

El primero observaba cómo el sujeto agresor era felicitado efusivamente por otro adulto. Un segundo grupo observaba cómo el sujeto agresor era duramente castigado y reprendido por el mismo personaje adulto a causa de lo que había hecho y terminaba soportando la misma agresión que había descargado contra el muñeco. El tercer grupo sólo se quedó con la primera parte; es decir, el sujeto agresor no recibía ni recompensa ni castigo. Luego, los tres grupos ingresaron a una sala de juegos acondicionada a semejanza de la que habían observado en la película y en la que se hallaba el muñeco agredido. De más está decir que los niños y las niñas que más respuestas agresivas expresaron fueron los del primer grupo; pero, cuando los investigadores incentivaron a que contasen lo que habían visto y oído, todos los niños y las niñas de todos los grupos demostraron haber aprendido cognoscitivamente los comportamientos violentos observados animándose a imitar al modelo agresor de la película. Este experimento llevó a la conclusión que las conductas violentas se aprenden siempre con independencia del tratamiento posterior que reciban y se expresan cuando son incentivadas (De Andrés 2006).

Es de resaltar que los niños y las niñas expresaron su conducta agresiva con el dominguillo sin que hubiera un refuerzo que motivara a la ejecución de dicha conducta. Es por ello que uno de los principios de la teoría del aprendizaje social cognitivo es que no se imita la conducta del modelo por la conducta misma, sino por la aceptación social de la misma.

2 Según el Diccionario Manual e Ilustrado de la Lengua Española (1981), dominguillo es el «muñeco que lleva un contrapeso en la base, y que, movido en cualquier dirección, vuelve siempre a quedar derecho". 
En este caso, los niños y las niñas imitan la conducta del modelo porque al repetirla obtienen los mismos beneficios positivos otorgados al modelo. En esto consiste el aprendizaje por la observación o modelado; en el que, si bien el entorno causa el comportamiento, la conducta también causa el ambiente. Este concepto fue definido, según Boeree (1998: 4) como «determinismo recíproco: el mundo y el comportamiento de una persona se causan mutuamente» ${ }^{3}$. [Traducción del autor del ensayo].

En este aspecto, el mismo Bandura (2006: 8 y 9) afirmó que: «Analizamos que el modelado social funciona mediante cuatro subfunciones que comprenden procesos de atención, representación, traducción a la ejecución y motivación». ¿Cuál es el contenido de estas cuatro subfunciones?

1. Atención. Si un sujeto va a aprender algo, requiere que esté atento. En consecuencia, todo aquello que interfiera en la atención como el deterioro o desequilibrio en el estado de salud o un estímulo en competencia intervendrá negativamente en el aprendizaje. Asimismo, las propiedades [colorido, dramatismo, atractivo, prestigio, competencia] del modelo influyen sobre la atención del observador. Una observación especial es que si el modelo se parece más al observador, se prestará más atención.

Este conjunto de variables condujo a Bandura hacia el análisis de la televisión y sus efectos sobre los niños. En su biografía ha narrado cómo se enfrentó a los grandes grupos de poder de los medios de comunicación norteamericanos llevando el tema hasta el Congreso de los Estados Unidos.

3 He labeled this concept reciprocal determinism: The world and person's behavior cause each other. 
2. Retención. Una vez captada la información por el sujeto, éste debe ser capaz de retener [recordar] aquello a lo que le ha prestado atención. Aquí es donde la imaginación y el lenguaje entran en juego; lo observado al modelo es almacenado en forma de imágenes mentales o descripciones verbales. Una vez almacenada la información, el sujeto puede evocar la imagen o describirla de manera que pueda reproducirla con su propia conducta.

3. Reproducción. Es la subfunción por la que las imágenes o descripciones guardadas deben ser traducidas a comportamientos. Para ello, el sujeto debe ser capaz de reproducir el comportamiento. El espectador puede observar una determinada conducta, pero, puede no ser capaz de reproducirla porque carece de experiencia previa. Sin embargo, si tengo experiencia previa de la conducta esta podrá mejorar al observar a un modelo. A modo de ejemplo: puedo observar a un bailarín profesional de ballet, pero no ser capaz de reproducir sus movimientos porque no sé bailar; pero, si tengo experiencia previa, mi observación me conducirá a mejorar mi conducta, la misma que será mejorada en la medida que la imagino ejecutándola y la practico. Al respecto, Iacoboni (2009), eminente neurocientífico, señala: «Antes de la investigación neurocientífica, sabíamos por los estudios psicológicos que imitar es una buena manera de aprender, mejor que probar por el método ensayo - error, trato de mirar a quien hace algo y aprendo observando».

4. Motivación. Cumplidos los requisitos anteriores no se hará nada, a menos que el sujeto no esté motivado a imitar; es decir, a menos que tenga buenas razones para hacerlo. ¿Qué motivos intervienen no como causantes del aprendizaje sino como impulsores del mismo? Refuerzos pasados, refuerzos prometidos, refuerzo vicario; asimismo, existen motivaciones negativas que impulsan a no imitar. A saber: castigo pasado, castigo prometido (amenazas) y castigo vicario. 
Como se puede apreciar, la teoría del aprendizaje cognitivo social toma en consideración tres aspectos: la conducta [acción], los procesos internos [procesos cognitivos, biológicos, psicológicos y afectivos] y los factores ambientales [físicos y sociales]. Es así que, Minici et al. (2008: 7 y 8), citando a Bandura, han señalado lo siguiente:

En su libro, Pensamiento y Acción (1986), lo expresa claramente: “...Dentro del modelo de reciprocidad triádica, la acción, la cognición y los factores ambientales actúan juntos para producir los cambios psicológicos. Las personas con problemas psicológicos crean una realidad social perturbadora como consecuencia de su comportamiento y de su interpretación errónea de los acontecimientos que se producen en su vida diaria. Por tanto, la contribución causal de la cognición se comprende y utiliza mejor en concierto con los demás interactuantes conductuales y ambientales. Las influencias psicológicas no son ni exclusivamente cognitivas ni conductuales (...), actúan bidireccionalmente para moldear el curso del cambio personal y ambiental"... 
Lo expuesto se podría representar gráficamente de la manera siguiente:

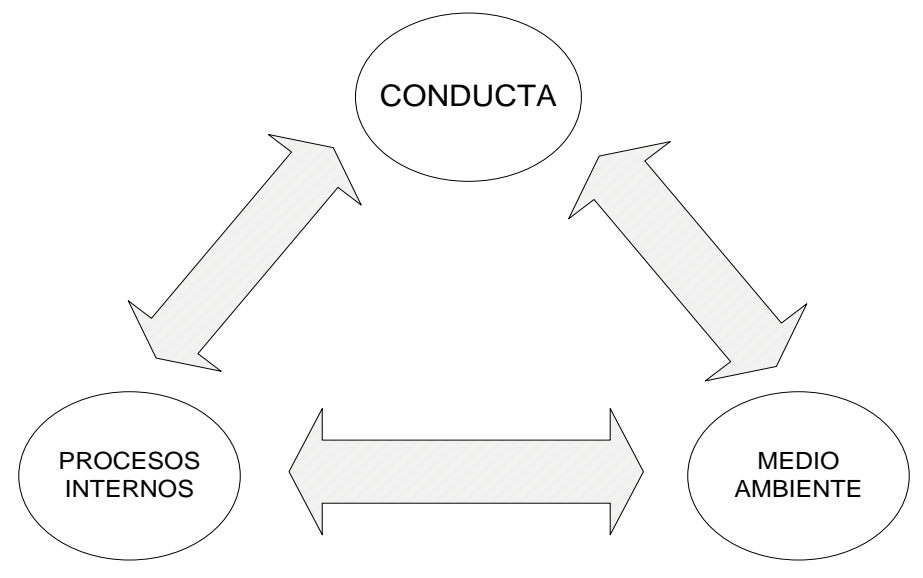

Noboa y Serrano-García (2006: 23) han afirmado que: «Bandura reconoce las distintas capacidades cognitivas que organizan el comportamiento del ser humano. Entre estas capacidades menciona: (a) la capacidad de simbolizar, (b) la capacidad vicaria, (c) la capacidad de planificar, (d) la capacidad autorreguladora, y (e) la reflexiva».

Por la capacidad de simbolización, el ser humano combina una serie de comportamientos que le permiten construir distintas alternativas y resolver problemas, entendidos estos como conjunto de hechos o circunstancias que dificultan el logro de un determinado objetivo que presenta la vida diaria. Los símbolos permiten efectuar representaciones cognitivas de las experiencias; es decir, se establecen, entre otras, las relaciones causa-efecto.

Por la capacidad vicaria, se aprende observando; el espectador al observar un comportamiento efectúa una representación mental de lo observado y experimenta en su interior lo que el modelo ejecuta y, por lo 
tanto, los efectos deseables del comportamiento del modelo son los que le proporcionan suficiente incentivo para imitar la ejecución. Por otro lado, si el observador toma conocimiento de las consecuencias negativas, en ocasiones, no realizará el comportamiento. Sobre el particular, es necesario señalar que no es una imitación pasiva sino una imitación eminentemente activa porque el observador ańade sus características personales y, por otro lado, el modelo puede ser real [padres, educadores, amigos], irreal [modelos mostrados a través de una película o de una telenovela], simbólico [imágenes a partir de fábulas, cuentos, mitos].

Por la capacidad de planificar, el ser humano dirige su actuar en forma anticipada previendo las consecuencias probables de sus acciones; es decir, las prevé en función a la consecución de determinadas metas y objetivos. Al ser representados los resultados simbólicamente en función de un logro futuro se convierten en motivación para la ejecución de lo previsto.

La capacidad autorreguladora se relaciona con el proceso de socialización y le permite al individuo el establecimiento de controles internos que dan dirección a su comportamiento. Dicha capacidad autorreguladora le permite al ser humano constituirse históricamente como persona y como miembro de una sociedad, de un colectivo; es decir, reconocerse unido a otros seres humanos bajo determinados parámetros. Cabe señalar que la capacidad autoreguladora se desarrolla y se construye y le permite a los seres humanos internalizar las demandas y las sanciones externas. El resultado es que el ser humano aprende a comportarse en sociedad.

Por la capacidad reflexiva, el ser humano no responde automáticamente a las demandas del entorno, sino que analiza su mundo interno, se conoce, así como el mundo representado simbólicamente en él, se evalúa y, de ser necesario, cambia su comportamiento. Por esta capacidad reflexiva, el ser humano actúa activamente frente al entorno. Es así que el mismo Bandura 
(2006: 9), en su autobiografía, responde a los argumentos contrarios al aprendizaje cognitivo social:

Una de estas falsas concepciones era que el modelado podía producir solamente reproducciones miméticas. Esta falsa concepción se eliminó evidenciando que el modelado implicaba abstraer la información trasmitida por los modelos específicos acerca de las estructuras y los principios subyacentes que gobiernan la conducta, en vez de una simple respuesta mimética de los modelos específicos [Bandura, 1986; Bandura y Zimmerman, 1978]. Una vez que el individuo aprende los principios guía, puede utilizarlos para generar versiones nuevas de la conducta que trascienden lo que ha sido visto u oído.

Es en este orden de ideas que es pertinente señalar que interviene un concepto fundamental de la teoría psicológica formulada por Bandura y es el de autoeficacia. Si bien este concepto no es relevante para los efectos del presente ensayo, se considera oportuno señalarlo con el propósito de no sesgar la exposición que se viene realizando. ¿Qué entiende Bandura por autoeficacia? El mismo Bandura (2001: 1) responde: «La autoeficacia percibida hace referencia a las creencias de las personas acerca de sus propias capacidades para el logro de determinados resultados».

\section{EL SISTEMA DE LAS NEURONAS ESPEJO}

Una de las preguntas que los científicos de distintas especialidades se han planteado es la siguiente: ¿qué es lo que ha sucedido a lo largo del proceso de evolución que ha permitido que, según la tesis de Gazzaniga, el ser 
humano resulte un ser único y distinto de los animales aún de aquellos que más cerca se encuentran en dicho proceso? ¿qué es lo que le ha permitido al ser humano, desde sus primeras y rudimentarias creaciones culturales, llegar a dominar su mundo, en el sentido más amplio del término, y que cada día se lance a nuevos descubrimientos? Gazzaniga (2010: 1) citando a Rakic señala: «El cerebro es el órgano que nos distingue de cualquier otra especie. Lo que nos hace diferentes no es la fuerza de nuestros músculos ni de nuestros huesos, es nuestro cerebro». Y el cerebro es el resultado de la confluencia dinámica de dos vertientes: la evolución biológica por selección natural y las representaciones interiores, producto de complejos procesos neurobiológicos y fisiológicos, que se han efectuado en la interacción con el medio ambiente. Dichas representaciones se han transmitido por vía genética y epigenética.

Las neuronas espejo, como ya se ha expresado anteriormente, fueron inicialmente ubicadas en la corteza ventral premotora [F5] y, posteriormente, en el lóbulo parietal inferior de los cerebros de los monos macacos.

Del Giudice et al., (2009: 350) señalan:

Las 'neuronas espejo' fueron descritas por primera vez en la corteza premotora de los monos macacos (Gallese, Fadiga, Fogassi y Rizzolatti, 1996; Keysers, Kohler, Umiltà, Nanetti, Fogassi y Gallese, 2003; Kohler, Keysers, Umiltà, Gallese Fogassi, y Rizzolatti, 2002) y tienen la propiedad de responder, tanto cuando el individuo realiza una acción como cuando ve o escucha 
a otra persona realizando una acción similar. ${ }^{4}$ (Traducción del autor del ensayo).

Según Keyser y Gazzola (2009, p.4):

En los primates, tres áreas del cerebro se han asociado particularmente con la percepción de las acciones de otros individuos: el surco temporal superior (STS), el área PF del lóbulo parietal inferior y el área F5 de la corteza premotora ventral [...] Se ha demostrado que dos de estas áreas, PF y F5, contienen las neuronas denominadas «neuronas espejo». Las neuronas espejo (NE) son una subclase de las neuronas visomotoras que tienen una peculiar combinación de propiedades de respuesta. ${ }^{5}$ (Traducción del autor del ensayo).

En el área F5 de los monos existen dos clases de neuronas: las neuronas canónicas que responden cuando se presentan objetos y las neuronas espejo que requieren una interacción entre un efector biológico (mano o boca) y un objeto. La sola percepción de un objeto o de una persona no produce ninguna activación de las neuronas espejo y estas reaccionan aunque sean

4 'Mirror neurons', first described in the premotor cortex of macaque monkeys (Gallese, Fadiga, Fogassi \& Rizzolatti, 1996; Keysers, Kohler, Umiltà, Nanetti, Fogassi \& Gallese, 2003; Kohler, Keysers, Umiltà, Fogassi, Gallese \& Rizzolatti, 2002) have the property of responding both while the individual executes an action and while he/she sees or hears another individual performing a similar action.

5 In primates, three brain areas have been particularly associated with the perception of the actions of other individuals: the superior temporal sulcus (STS), area PF of the inferior parietal lobule, and area F5 of the ventral premotor cortex (see Fig. 1 left). Two of these areas, PF and F5, have been shown to contain neurons called 'mirror neurons.' Mirror neurons $(\mathrm{MN})$ are a subclass of visuo-motor neurons that have a peculiar combination of response properties. 
eventualmente recompensadas. Asimismo, un aspecto que es importante resaltar es la relación entre las propiedades visuales y motoras de las neuronas espejo; algunas de estas que responden ante la observación de acciones que tienen un propósito, por ejemplo aprehender un objeto, y el uso de medios para llevar a cabo la acción, por ejemplo aprehensión con precisión, se las denomina estrictamente congruentes y constituyen la tercera parte de las neuronas espejo. Y a las que no requieren de la observación de la misma acción motora se les denomina generalmente congruentes y constituyen las dos terceras partes del sistema. (Rizzolatti y Craighero, 2004). Al respecto, Soto y Vega (2007:50) afirman que las neuronas espejo son bimodales: visuales y motoras porque «no solo responden a los movimientos de otros, sino que participan en nuestros propios movimientos». Además señalan que este hallazgo rompe la categorización tradicional de las neuronas; es decir:

[...] no son ni puramente motoras ni puramente sensoriales, sino ambas a la vez. De hecho parte de la idea que se tiene hoy es que juegan un papel relevante en la capacidad de los primates y del hombre para comprender de forma casi inmediata los movimientos, las acciones y, eventualmente, las intenciones de otros sujetos. Cerebro que actúa es cerebro que comprende. Se trata, como han establecido Rizzolati y sus colaboradores, de una comprensión pragmática, preconceptual y prelingüística. 'Vemos porque actuamos y podemos actuar precisamente porque vemos'. 


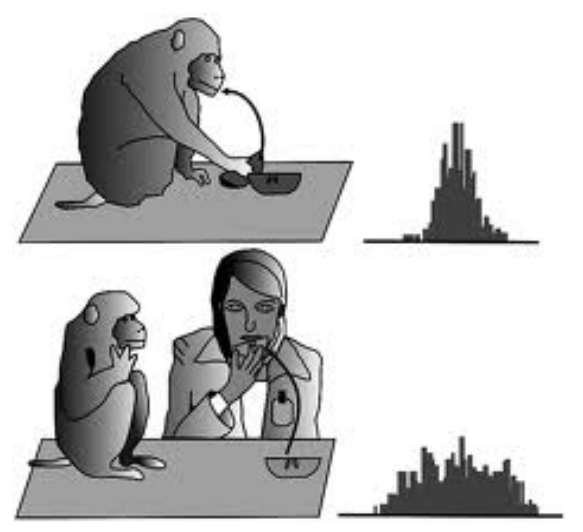

Figura 1. En la parte superior se esquematiza a un primate que toma un objeto y se lo lleva a la boca y abajo el primate observa a una persona realizar esa misma acción. A la derecha los histogramas muestran la intensidad de las descargas de las neuronas del área F-5 de la corteza cerebral del primate. Puede observarse que la actividad de estas neuronas es muy similar cuando el primate realiza la acción de tomar el objeto que cuando solo la observa. Soto y Vega (2007:52).

Cabe destacar, que los hallazgos científicos obtenidos a partir de experimentos con animales no se pueden extrapolar mecánicamente a los seres humanos; por ello, el sistema de neuronas espejo en los seres humanos difiere cualitativamente del de los monos porque según Rizzolatti citado por Van Gog et al. (2009:23) «Se supone que los monos pueden tratar de imitar porque sólo entienden el objetivo de una acción; pero es de suponer que no pueden codificar los detalles de la acción con la que se logra el objetivo. Los seres humanos pueden codificar cómo se alcanza el objetivo, permitiendo así 
la imitación ${ }^{6} »$. (Traducción del autor del ensayo).

¿Cómo está constituido el sistema de neuronas espejo en los seres humanos? Rizzolatti y Craighero (2004:176) señalan lo siguiente:

Un gran número de estudios han demostrado que la observación de las acciones realizadas por otros activa en los seres humanos una compleja red formada por los lóbulos occipital, temporal y parietal, áreas visuales, y dos regiones corticales, cuya función es fundamentalmente o predominantemente motora [...]. Estas dos últimas regiones son la parte rostral del lóbulo parietal inferior y la parte inferior de la circunvolución precentral más la parte posterior de la circunvolución frontal inferior (IFG). Estas regiones forman el núcleo del sistema humano de neuronas espejo. ${ }^{7}$ (Traducción del autor del ensayo).

El sistema de las neuronas espejo también se encuentra profusamente en el Área de Broca lo que según sostienen Soto y Vega (2007:52) « [...] podría indicar su participación en la adquisición del lenguaje contribuyendo con un sistema capaz de imitar complejos patrones de movimiento de la boca». En este aspecto, así como hay neuronas bimodales motoras y sensoriales con respecto al tacto, también existen neuronas bimodales

$6 \quad$ It is assumed that monkeys can therefore only understand the goal of an action and try to copy it, but they presumably cannot encode the details of the action with which the goal is achieved. Humans can encode how the goal is achieved, thereby enabling imitation.

7 A large number of studies showed that the observation of actions done by others activates in humans a complex network formed by occipital, temporal, and parietal visual areas, and two cortical regions whose function is fundamentally or predominantly motor... These two last regions are the rostral part of the inferior parietal lobule and the lower part of the precentral gyrus plus the posterior part of the inferior frontal gyrus (IFG). These regions form the core of the human mirror-neuron system. 
motoras y sensoriales pero auditivas, lo que contribuiría a la programación de movimientos conducentes a sonidos.

Es aceptado que la supervivencia y el éxito del ser humano radica en el manejo de sus interacciones sociales; es decir, interpretar las acciones de los que lo rodean y de aquellos con los que tiene que interactuar directa o indirectamente. Un aspecto que merece atención es ¡cómo se construye dentro de este interactuar del ser humano el sentimiento de pertenencia a un colectivo [familia, barrio, escuela, región, nación]? Una primera aproximación la presenta Gallese (2009: 520) cuando propone que:

[...] la identificación social incorpora los ámbitos de acción, sensaciones, afectos y emociones y está sustentada en la activación de circuitos neuronales [...]. Cuando se observa directamente actuar a otros individuos con toda su fuerza expresiva (el modo cómo actúan, las emociones y sentimientos que ellos ponen de manifiesto) se establece en forma automática e interiormente un lazo interpersonal significativo. El descubrimiento de las neuronas espejo y de otros mecanismos espejo en el cerebro humano muestra que los mismos circuitos son activados cuando los actos expresivos son ejecutados y percibidos. En consecuencia, neuronalmente estamos constituidos para conformar el espacio nosotros. ${ }^{8}$ (Traducción del autor del ensayo).

8 The hypothesis being proposed here is that social identification incorporates the domains of action, sensations, affect, and emotions and is underpinned by the activation of shared neural circuits... When observing other acting individuals, and facing their full range of expressive power (the way they act, the emotions and feelings they display), a meaningful embodied interpersonal link is automatically established.

The discovery of mirror neurons and of other mirroring mechanisms in the human brain shows that the very same neural substrates are activated when these expressive acts are both executed and perceived. When observing other acting individuals, and facing their full range of expressive power (the way they act, the emotions and feelings they display), a meaningful embodied interpersonal link is automatically established. Thus, we have a neutrally instantiated we-centric space. 
La interacción social, que se ha comentado, sería muy poco probable si el ser humano no estuviera en capacidad de aprehender [entender, comprender y sentir] la intencionalidad y emoción del otro, ya sea considerado como individuo o como colectivo. Como se ha señalado anteriormente, las neuronas espejo del sistema ubicadas en el área premotora del cerebro cumplen también una función fundamental que es comprender la conducta de los otros. Esta comprensión implica deducir, a partir de determinados indicativos previamente experimentados por el observador, el propósito, intencionalidad $\mathrm{u}$ objetivo de la conducta observada y además sentir emocionalmente lo que siente el otro; es lo que se define como empatía. Ahora bien, la empatía tiene dos componentes esenciales: el cognitivo y el emocional. ¿Actúan como un sistema único o son independientes? Al respecto las evidencias científicas no han podido establecer una respuesta uniforme; pero, según Moya (2010: 92) lo que sí se ha establecido es que:

[...] el área correspondiente con la parte opercular del giro frontal inferior (área 44 de Brodmann) mostró ser esencial para la empatía emocional, mientras que las áreas que comprenden las partes anteriores del giro frontal superior y medio, y del giro orbital, el recto y la zona más anterior del giro superior frontal (áreas 10 y 11 de Brodmann, respectivamente) lo fueron para la cognitiva.

Pero, ¿por qué unos sujetos reaccionan con mayor empatía que otros? Entre otras razones porque su sistema de neuronas espejo muestra mayor activación.

¿Qué sucede en aquellos seres humanos que muestran una alteración en las interacciones sociales con otros seres humanos, reducida comunicación verbal y no verbal y un repertorio de actividades e intereses característicamente 
restringidos? Es decir ¿qué sucede en los mecanismos neurofisiológicos de las personas cuya conducta corresponde a las condiciones del espectro autista? Sobre el particular se ha establecido, mediante datos encefalográficos, que en sujetos que no presentan dichas condiciones al realizar u observar la ejecución de actos motores presentan una supresión del ritmo $\mathrm{mu}^{9}$ en las áreas sensoriomotoras de la región premotora lo cual se correlaciona con la activación del sistema de neuronas espejo. En cambio, en las mismas condiciones establecidas, los niños que presentan las condiciones del espectro autista no muestran dicha supresión del ritmo mu, lo cual conduce a inferir que el sistema de neuronas espejo no se activa normalmente lo que conduce a esa incapacidad del reconocimiento empático de las conductas de los demás. (Cornelio, 2009). Palau (2011: 144) afirma que: «Esta ausencia de supresión mu permanece a lo largo de la vida».

El postulado subyacente a todo lo expuesto es: la conducta humana es resultado de la interacción dinámica de tres condiciones esenciales: la genética, el sistema nervioso y el ambiente. Bajo esta perspectiva se puede afirmar que es multideterminada y ninguna de las condiciones anteriormente señaladas por sí sola puede explicar la conducta humana. Tal vez un ejemplo podría graficar lo señalado: las células de la retina cuya función es recoger, elaborar y transmitir las sensaciones visuales son heredadas genéticamente y el proceso de síntesis de proteínas también es heredado genéticamente; pero, no se desarrollan si no hay interacción con la luz. La interrelación de esos dos elementos lo genético y el medio ponen en acción esa función neuronal.

9 Registro electroencefalográfico que se observa en estado de reposo y con los ojos cerrados con frecuencia dominante en la banda $8-13 \mathrm{hz}$; es el reflejo de la sincronía en las neuronas corticales de la corteza sensoriomotora. (Palau-Baduell et al., 2011). 
En el esfuerzo por comprender y explicar el aprendizaje humano Bandura formula su teoría psicológica del aprendizaje cognitivo social cuyo eje principal es que el ser humano aprende, principalmente, por imitación. El observador replicará la acción observada por las consecuencias positivas que han recaído en el sujeto observado. Si bien fue un intento muy loable para explicar el aprendizaje humano, este quedaba a nivel de constructo. ¿Cómo se podía explicar científicamente? ¿Cuál era el fundamento neurofisiológico de dicho constructo? Van Gog et al. (2009: 21) sostienen que:

El descubrimiento del sistema de las neuronas espejo es el mayor hallazgo relevante al aprendizaje por observación. Este sistema está pensado para que juegue un rol importante en la comprensión de las acciones realizadas por otros y puede ser responsable de nuestra habilidad para aprender observando e imitando a otros». ${ }^{10}$ (Traducción del autor del ensayo).

En el aspecto educativo, entendido este, en la acepción más comprensiva del concepto, como el proceso mediante el cual un sujeto además de adoptar las normas, valores, usos y costumbres de una sociedad concreta, no sólo local sino universal, prepara sus aptitudes y actitudes para recrear esa cultura, lo seńalado tiene una enorme repercusión por cuanto si el ser humano aprende por imitación porque dispone genéticamente de los procesos para dicho aprendizaje, la acción, voluntaria o no, metodológicamente programada o de manera informal, que ejercen los educadores [padres, maestros, la sociedad en conjunto] sobre los sujetos en formación es fundamental y de ella dependerá el futuro de las generaciones.

10 The discovery of the mirror neuron system is a major neuroscience finding relevant to observational learning. This system is thought to play an important role in the understanding of actions made by others and may be responsible for our ability to learn by observing and imitating others. 
Al respecto Rizzolati (2005) señaló lo siguiente:

El mensaje más importante de las neuronas espejo es que demuestran que verdaderamente somos seres sociales. La sociedad, la familia y la comunidad son valores realmente innatos. Ahora, nuestra sociedad intenta negarlo y por eso los jóvenes están tan descontentos, porque no crean lazos. Ocurre algo similar con la imitación, en Occidente está muy mal vista y sin embargo, es la base de la cultura. Se dice: «No imites, tienes que ser original», pero es un error. Primero tienes que imitar y después puedes ser original. Para comprenderlo no hay más que fijarse en los grandes pintores.

En este aspecto, cabe dirigir la atención a las tecnologías de información y comunicación. Es conocido que, muchas veces, sus contenidos, sus mensajes explícitos o subliminales son violentos y se proporcionan a cerebros en formación, lo cual progresivamente va modelando conductas agresivas y que muchas veces tienen el reconocimiento social. ¿Cómo se puede formar conciencias para una cultura de paz? Tal vez se manifieste que no se puede ocultar la realidad; sí, es cierto, pero ¿por qué magnificarla más de lo que realmente es?

Para el caso de sujetos que se encuentran en las condiciones del espectro autista, lo expuesto permite extraer, también, consideraciones importantes: son cerebros diferentes cuyo ritmo y contenido de aprendizaje es diferente y es preciso conocerlo, respetarlo y brindarle las condiciones externas adecuadas. $Y$ esa diferencia debe ser considerada al momento de los distintos aprendizajes apelando a un principio fundamental de las neurociencias: la plasticidad neuronal. 
No se desea concluir este ensayo sin señalar que psicología y neurociencia son dos ciencias distintas, cada una con su objeto de estudio claramente establecido. No se trata de subordinar una a otra, sino a partir de descubrimientos científicos reforzar los constructos establecidos y llegar a una mejor explicación del comportamiento humano. 


\section{BIBLIOGRAFÍA}

BANDURA, Albert

2001 Guía para la construcción de escalas de Autoeficacia. (Revisada en marzo, 2001), «http://des.emory.edu/mfp/effguideSpanish.html». Traducción de Fabián Olaz; María Inés Silva; Edgardo Pérez. Universidad Nacional de Córdoba. Argentina. Consulta hecha en 03/05/2011.

Bandura, Albert

2006 Autobiografía de Albert Bandura. A history of psychology in Autobiography. (Vol. IX). «http://www.des.emory.edu/mfp/ BanduraAutoSpanish.pdf» Washington DC: American Psychological Association. Traducción de Eugenio Garrido. Consulta hecha en $5 / 04 / 2011$.

Boeree, George

1998 Albert Bandura 1925 - present. Teorías de la Personalidad.. «http://2 09.85.62.24/28098/188/0/p373518/pt_bandura.pdf». Consulta hecha en $3 / 05 / 2011$.

Cornelioo-Nieto, José

2009 Autismo infantil y neuronas en espejo. Revista de Neurología. No 48, (Supl 2), S27-29. «http://www.neurologia.com/pdf/Web/48S02/ bbS02S027.pdf» Consulta hecha en 31/05/2011. 
De ANDrÉs, Tomás

2006 El Desarrollo de la Inteligencia Fúlmica. La comprensión audiovisual y su evolución en la infancia y la adolescencia. Centro Nacional de Información y Comunicación Educativa. «http://ares.cnice.mec. es/informes/15/index.htm». Ministerio de Educación y Ciencia. Informe No 15. Madrid. Consulta hecha en 16/04/2011.

Del Giuduce, Marco; Valeria Manera y Christian Keysers

2009 Programmed to learn? The ontogeny of mirror neurons. Developmental Science, 12:2, 350-363. «http://www.psych.unito. it/csc/pers/delgiudice/pdf/DelGiudice_etal_2009_ontogeny_mirrorneurons_ds.pdf». Consulta hecha en 9/05/2011.

Garrido, Eugenio

2000 Albert Bandura: Voluntad Cientifica. «http://des.emory.edu/mfp/ BanduraGarrido.PDF». Consulta hecha en 10/04/2011.

Gazzaniga, Michael

2010 ¿Qué nos hace humanos?. «http://www.planetadelibros.com/pdf/primer_ gazzaniga.pdf». Consulta hecha en 9/05/2011.

\section{Gallese, Vittorio}

2009 Mirror neurons, embodied simulation and the neural basis of social identification. Psychoanalytic Dialogues. No 19, 519-536. «http:// www.unipr.it/arpa/mirror/pubs/pdffiles/Gallese/Gallese\%20PD\%20 2009a.pdf». Consulta hecha en 30/05/2011. 
IACOBONI, Marco

2009 Las neuronas espejo demuestran que nuestro cerebro está 'conectado para empatizar'. Entrevista en video divulgada por la Universidad Nacional de Educación a Distancia. Madrid. «http://www. agenciasinc.es/esl/videos/detalle/39813». Consulta hecha en 03/05/2011.

2010 Mentes conectadas sin brujería. Entrevista concedida al programa Redes No 56 de la Radio Televisión Española el 18 de abril de 2010. «http://www.rtve.es/alacarta/videos/redes/redes-mentes-conectadas-sinbrujeria/748264/». Consulta en 04/05/2011.

Keysers, Christian y Valeria Gazzola

2009 Unifying Social Cognition. En Jaime Pineda (editor), Myrror Neurons System. The role of Mirroring Processes in Social Cognition, 3-38. New York: Human Press.

Martin-Loeches, Manuel; María del Pilar Casado y Alejandra Sel 2008 La evolución del cerebro en el género homo: la neurobiología que nos hace diferentes. Revista de Neurología, Volumen 46 (12). (pp. 731-741). «http://www.ucm.es/info/neurosci/docs/z120731.pdf». Consulta hecha en 12/05/2011. 
Minici, Ariel; Carmela Rivadeneira y José DaHab

2008 Distorsiones cognitivas: su relación con los paradigmas de condicionamiento clásico y operante. Revista de Terapia Cognitiva Conductual. No 15. «http://www.cognitivoconductual.org/revista/ distorsiones-cognitivas/». Consulta hecha en 18/04/2011.

Moya-Albiol, Luis; Neus Herrero y M. Consuelo Bernal

2010 Bases neuronales de la empatía, Revista de Neurología 2010, No 50, 89-100. «http://www.neurologia.com/pdf/Web/5002/bd020089.pdf» Consulta hecha en 31/05/2011.

Mukamel, Roy; Arne Ekstrom; Jonas Kaplan; Marco Iacoboni y Itzhak FRIED

2010 Single-Neuron Responses in Humans during Execution and Observation of Actions. Current Biology. Vol. 20, No 8, 750-756. «http://www.sciencedirect.com/science?_ob=MImg\&_imagekey=B6VRT4YT72MH-1-C\&_cdi=6243\&_user=10\&_pii=S0960982210002332\&_ origin=search \&_zone=rslt_list_item $\&$ _coverDate=04\%2F27\%2F2010\&_ sk=999799991 \&wchp=dGLzVzz-zSkzk\&md5=6673590063b45324fa62 00bb2bf22aad\&ie=/sdarticle.pdf». Consulta hecha en 28/04/2011.

Noboa, Patricia e Irma Serrano-García

2006 Autoeficacia en la negociacion sexual: retos para la prevención de VIH/SIDA en mujeres Puertorriqueñas. Revista Latinoamericana de Psicología. Vol. 38. No 001. «http://www.accessmylibrary. com/article-1G1-153413955/autoeficacia-en-la-negociacion. htmlhttp://redalyc.uaemex.mx/pdf/805/80538102.pdf». Consulta hecha en 28/04/2011. 
Palau-Baduell, Monserrat; Antonio Valls-Santasusana y Berta SalvadóSALVAdó

2011 Trastornos del espectro autista y ritmo mu. Una nueva perspectiva neurofisiológica. Revista de Neurología, Vol. 52 (Supl. 1), S141-S146. "http://www.neurologia.com/pdf/Web/52S01/bfS01S141. pdf» Consulta hecha en 31/05/2011.

Real Academia Española

1981 Diccionario Manual e Ilustrado de la Lengua Española. EspasaCalpe. S.A. Madrid. España.

Rizzolatti, Giacomo y Laila Craighero

2004 The mirror-neuron system. Annual Review of Neuroscience, Vol. 27, 169 - 192. «http://www.annualreviews.org/doi/full/10.1146/annurev. neuro.27.070203.144230». Consulta hecha en 27/05/2011.

Rizzolati, Giacomo

2005 Las neuronas espejo te ponen en el lugar del otro. Entrevista realizada por Ángela Boto y publicada en EL PAÍS.COM el 19 de octubre de 2005. «http://www.elpais.com/articulo/futuro/neuronas/espejo/ ponen/lugar/elpfutpor/20051019elpepifut_6/Tes». Consulta hecha en $19 / 05 / 2011$.

Rondal, Jean Adolphe

2007 Teoría de la mente y lenguaje. Revista de Logopedia, Foniatría y Audiología. Vol. 27. № 2, 51-55. «http://www.elsevier.es/sites/default/ files/elsevier/pdf/309/309v27n02a13153289pdf001.pdf». Consulta hecha en $28 / 04 / 2011$. 
Soto, Enrique y Rosario Vega

2007 El sistema de neuronas en espejo. Elementos de Ciencia y Cultura. Año/volumen 14. No 068, 49-53. «http://redalyc.uaemex.mx/ pdf/294/29406807.pdf». Consulta hecha en 17/05/2011.

Van Gog, Tamara; Fred Pass; Nadine Marcus; Paul Ayres y John Sweller 2009 The Mirror Neuron System and Observational Learning: Implications for the Effectiveness of Dynamic Visualizations. Educational Psychology Review. (on line). Volume 21, No 1, pp. 2130. «http://dspace.learningnetworks.org/bitstream/1820/1858/1/VanGogetal_EPR_2009.pdf». Consulta en 21/05/2011. 